\title{
Organization of Block Copolymers using NanoImprint Lithography: Comparison of Theory and Experiments
}

\author{
Xingkun Man ${ }^{1}$, David Andelman ${ }^{1, *}$, Henri Orland ${ }^{2}$, Pascal Thébault ${ }^{3}$, \\ Pang-Hung Liu ${ }^{3}$, Patrick Guenoun ${ }^{3}$, Jean Daillant ${ }^{3}$, Stefan Landis ${ }^{4}$ \\ ${ }^{1}$ Raymond and Beverly Sackler School of Physics and Astronomy, \\ Tel Aviv University, Ramat Aviv 69978, Tel Aviv, Israel \\ ${ }^{2}$ Institut de Physique Théorique, CE-Saclay, \\ F-91191 Gif-sur-Yvette Cedex, France \\ ${ }^{3}$ IRAMIS, LIONS, UMR SIS2M 3299 CEA-CNRS, \\ CEA-Saclay, F-91191 Gif-sur-Yvette Cedex, France \\ ${ }^{4}$ CEA, LETI, Minatec, 17 rue des martyrs, \\ F-38054, Grenoble Cedex 9, France
}

(Dated: Jan 2, 2011)

\begin{abstract}
We present NanoImprint lithography experiments and modeling of thin films of block copolymers (BCP). The NanoImprint technique is found to be an efficient tool not only to align lamellar phases perpendicularly to the substrate, but also to get rid of in-plane defects over distances much larger than the natural lamellar periodicity. The modeling relies on self-consistent field calculations done in two- and three-dimensions, and is found to be in good agreement with the experiments. It also offers some insight on the NanoImprint lithography setup and on the conditions required to perfectly ordered BCP lamellae.
\end{abstract}




\section{INTRODUCTION}

One of the main challenges of contemporary design of microchips is to find affordable techniques of patterning silicon wafers at the nanoscopic level [1]. To address this question, self-assembling block copolymers (BCP) have been suggested as potent candidates to provide patterns for nanolithography [2, 3]. The aim is to produce thin and structured BCP films with patterns such as lamellae, cylinders and spheres [4] that can then be transferred to a substrate.

When thin films of BCP are cast on a surface, they self-assemble into one of several possible nanostructures having a specific orientation with respect to the substrate. In particular, by adjusting the surface interactions and film thickness, it is possible to produce lamellar and cylindrical phases in an orientation perpendicular $[5,6]$ to the substrate. However, while lamellar and cylindrical phases can be perpendicularly aligned on a large scale [7, 8], the ever-remaining challenge for micro-electronic applications is to find affordable and efficient techniques for in-plane organization, with minimal amount of defects. This will allow producing devices that are hundreds of micrometers in size, where precise spatial accessibility is required.

Several attempts have been made to address this challenge. They include, among others, chemical patterning of the substrate by e-beam lithography $[9,10]$ or by prepositioning of another copolymer layer [11], and graphoepitaxy [12], where an artificial surface topography of grooves separated by walls is created on the substrate. Due to such topographical constraints, ordered regions of BCP are obtained over length scales of micrometers [12-15].

A more recent technique addressing the same issue is the nanoimprint lithography (NIL)[16-19], and it has potential advantages in terms of cost and simplicity. It uses surface micrometer-sized structural features of a reusable mold made by standard lithographic techniques to guide the self-assembly of the BCP at the nanometer scale. In Ref. [18] a cylindrical phase oriented perpendicularly to the substrate was imprinted by NIL where the NIL patterns are about twice or three times larger than the BCP period. The resulting BCP phase is aligned perpendicularly, but the imprint procedure is found to induce unwanted de-

fects. In another attempt [19], alignment of lamellae was produced by NIL, but the lamellae were not oriented perpendicular to the substrate.

Motivated by previous studies, we attempt in this paper to improve on the NIL procedure. In particular, we focus on NIL setups that produce perpendicular aligned lamellae with no defects, even when the NIL structural features are much larger than the BCP period. 


\section{MATERIALS AND METHODS}

The symmetric di-block copolymer polystyrene-b-polymethylmethacrylate $\left(\mathrm{PS}_{52 \mathrm{~K}}-\mathrm{b}-\right.$ PMMA $_{52 \mathrm{~K}}$, PDI:1.09) was purchased from Polymer Source Inc and exhibits a lamellar phase of period $\ell_{0}=49 \mathrm{~nm}$ in the bulk [10]. PS and PMMA blocks share very similar values of surface tensions, in the range of $29.7 \mathrm{mN} / \mathrm{m}-29.9 \mathrm{mN} / \mathrm{m}$ and $29.9 \mathrm{mN} / \mathrm{m}-31 \mathrm{mN} / \mathrm{m}$, respectively, at the temperatures used for film annealing $\left(\sim 170^{\circ} \mathrm{C}\right)$. The glass transition temperature of $\mathrm{PS}$ and $\mathrm{PMMA}$ is $100^{\circ} \mathrm{C}$ and $105^{\circ} \mathrm{C}$, respectively.

UV/ozone apparatus was used both for wafer cleaning and octadecyl-trichlorosilane (OTS) self-assembly monolayer (SAM) oxidation. The wafers were cleaned by irradiation before the silanization treatment. The OTS SAM oxidation was performed as described in Ref. [6] in order to reach a precise surface energy. To get a better contrast during AFM imaging, selective phase etching of PMMA can be applied just before the imaging. To avoid ozone formation that would degrade both the PS and PMMA blocks, the reaction chamber was flushed for $30 \mathrm{~min}$ with nitrogen before and during UV-irradiation. Samples were then irradiated for $30 \mathrm{~min}$ and subsequently treated with glacial acetic acid during $1 \mathrm{~h}$ to ensure complete removal of degraded PMMA fragments.

Solutions of $1 \mathrm{wt} \%$ of BCP in toluene were prepared and spin coated at $1800 \mathrm{rpm}$ onto silanized silicon wafers to produce BCP films with thickness of about $40 \mathrm{~nm}$ (below $\ell_{0}$ ). Subsequently, the samples were annealed in a vacuum oven at $170^{\circ} \mathrm{C}$ at a pressure of less than $3 \mathrm{KPa}$ for 1 day. The resulting lamellar phase was examined and found to be perpendicular to the neutral substrate but with many in-plane defects [6]. Then, the sample was treated by thermal NIL, which consists of embossing the thin BCP film, heated above its glass transition temperature, by a reusable mold made of a series of grooves as is shown schematically in Fig. 1(a).

Imprint experiments have been carried out at the CEA/LETI clean room in Grenoble, France, using a EVGß520HE press. The mold is a 4" silicon wafer with topographical features made of grooves of tens of nanometers in height with groove width $\left(\omega_{h}\right)$ and intergroove separation $\left(\omega_{l}\right)$ in the range of hundreds of nanometers [Fig. 1(b)]. The mold surface

is coated with a perfluorinated polymer layer to avoid BCP adhesion. An overall pressure of about $0.3 \mathrm{MPa}$ was applied to the $\mathrm{BCP}$ film, first at a temperature of $120^{\circ} \mathrm{C}$ during 7 hours, then at $170^{\circ} \mathrm{C}$ during 60 hours.

Surface imaging after imposing NIL on the BCP film was performed by SEM and AFM. SEM imaging was performed with a Hitachi 9300 apparatus, operated at optimal voltages of about $500 \mathrm{~V}$. This voltage allows observation of BCP phase organization, without PMMA etching, due to a smaller penetration depth of the electron beam and enhanced secondary electron emission yield. AFM imaging has been performed using a NanoScope V (Digital 


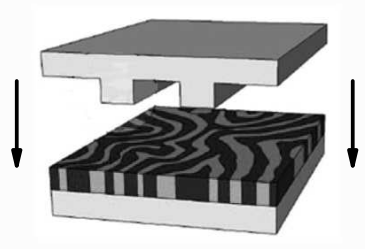

(a)

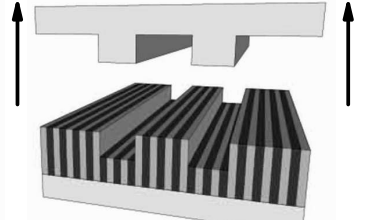

)

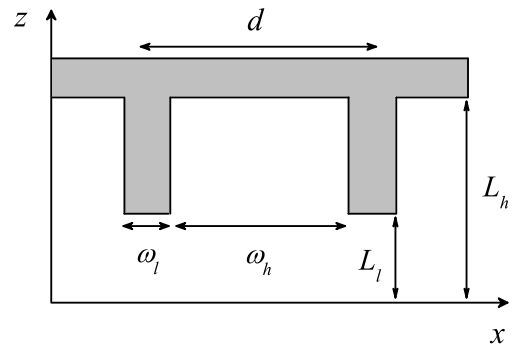

(b)

FIG. 1: The NIL setup. (a) A grooved top mold is pressed upon a BCP film oriented perpendicularly to a bottom substrate. The lamellae follow the direction of the mold grooves. (b) A cut (side view) through the modeled NIL setup consisting of a periodically patterned mold (top surface). The periodicity in the $x$-direction is $d=\omega_{l}+\omega_{h}$, with $\omega_{l}$ and $\omega_{h}$ being the finger width and groove width, respectively. The BCP film fills the gap between the two surfaces and its thickness varies between $L_{l}$ and $L_{h}$.

Instruments). The samples were analyzed using the AFM tapping mode in air and with silicon cantilevers of $125 \mu \mathrm{m}$ in length (Ultrasharp, Micro Masch). Their resonance frequency ranges between 265 and $400 \mathrm{KHz}$, whereas their force constant lies in between $20-75 \mathrm{~N} / \mathrm{m}$ and the tip radius of curvature is less than $10 \mathrm{~nm}$. The scan rate was chosen in order to obtain the best contrast in phase images and the least deviation between height trace and retrace scans.

\section{THE SELF-CONSISTENT FIELD THEORY}

We use self-consistent field (SCF) theory to investigate the behavior of a melt of A-B di-block copolymer (BCP) film at nano-patterned surfaces. The BCP film has $n$ polymer chains, each having a length $N=N_{A}+N_{B}$ in terms of the Kuhn length $a$, which is assumed, for simplicity, to be the same for the $A$ and $B$ monomers. Hence, the A-monomer molar fraction $f=N_{A} / N$ is equal to its volume fraction. In addition, hereafter we concentrate on symmetric di-BCP, $N_{A}=N_{B}$ having $f=0.5$. The symmetric BCP yields thermodynamically stable lamellar phases of periodicity $\ell_{0}$, as temperature is lowered below the order-disorder temperature (ODT). We rescale all lengths, $r \rightarrow r / \ell_{0}$, by the natural periodicity of the $\mathrm{BCP}, \ell_{0} \simeq 4.05 R_{g}$, where $R_{g}$ is the chain radius of gyration $R_{g}^{2}=N a^{2} / 6$. Similarly, the curvilinear coordinate along the chain contour, $s$, is rescaled by $N$, yielding $s \rightarrow s / N$. With these conventions, the free energy for such a BCP film confined between the two surfaces is 


$$
\begin{aligned}
\frac{N a^{2}}{\Omega_{1} \ell_{0}^{3}} \frac{F}{k_{B} T} & =\frac{F}{n k_{B} T}=\frac{1}{\Omega_{1}} \int \mathrm{d}^{3} r\left[\chi \phi_{A}(r) \phi_{B}(r)-\omega_{A}(r) \phi_{A}(r)-\omega_{B}(r) \phi_{B}(r)\right] \\
& -\ln Q_{C}-\frac{1}{\Omega_{1}} \int \mathrm{d}^{3} r\left[u_{A}(r) \phi_{A}(r)+u_{B}(r) \phi_{B}(r)\right] \\
& +\frac{1}{\Omega_{1}} \int \mathrm{d}^{3} r \eta(r)\left[\phi_{A}(r)+\phi_{B}(r)-1\right]
\end{aligned}
$$

The system has a total rescaled volume $\Omega_{1}$. The closed-packing density (monomer per unit area) is $\rho_{0}=a^{-3}$, and the Flory-Huggins parameter is $\chi$. The dimensionless volume fractions of the two components are defined as $\phi_{A}(r)$ and $\phi_{B}(r)$, respectively, whereas $\omega_{j}(r)$, $j=\mathrm{A}, \mathrm{B}$, are the auxiliary fields coupled with $\phi_{j}(r)$, and $Q_{C}$ is the single-chain partition function in the presence of the $\omega_{A}$ and $\omega_{B}$ fields. The third term represents a surface-energy preference, where $u_{A}$ and $u_{B}$ are the short-range interaction parameters of the surface with the $\mathrm{A}$ and $\mathrm{B}$ monomers, respectively. Formally, $u_{A}(r)$ and $u_{B}(r)$ are surface fields and have non-zero values only on the surface(s). It is worth noting that due to the above rescaling, the variables in Eq. (1) should also be rescaled by: $\chi \rightarrow N \chi, \omega_{j}(r) \rightarrow N \omega_{j}(r)$ and $u_{j}(r) \rightarrow N u_{j}(r)$.

Finally, the last term in Eq. (1) includes a Lagrange multiplier $\eta(r)$ introduced to ensure the incompressibility condition of the BCP melt:

$$
\phi_{A}(r)+\phi_{B}(r)=1 \quad \text { for all } r \in \Omega_{1}
$$

By inserting this condition, Eq. (2), in the surface free energy term of Eq. (1), the integrand becomes $u_{A} \phi_{A}+u_{B} \phi_{B}=\left(u_{A}-u_{B}\right) \phi_{A}+u_{B}$. Hence, $u(r) \equiv u_{A}(r)-u_{B}(r)$ is the only needed surface preference field to be employed hereafter.

Using the saddle-point approximation, we can obtain a set of self-consistent equations

$$
\begin{aligned}
\omega_{A}(r) & =\chi \phi_{B}(r)-u_{A}(r)+\eta(r) \\
\omega_{B}(r) & =\chi \phi_{A}(r)-u_{B}(r)+\eta(r) \\
\phi_{A}(r) & =\frac{1}{Q_{C}} \int_{0}^{f} \mathrm{~d} s q_{A}(r, s) q_{A}^{\dagger}(r, f-s) \\
\phi_{B}(r) & =\frac{1}{Q_{C}} \int_{0}^{1-f} \mathrm{~d} s q_{B}(r, s) q_{B}^{\dagger}(r, 1-f-s)
\end{aligned}
$$

where the incompressibility condition, Eq. (2), is obeyed, $f=N_{A} / N$ and the single-chain free energy $Q_{c}$ is:

$$
Q_{C}=\frac{1}{\Omega_{1}} \int \mathrm{d}^{3} r q_{A}^{\dagger}(r, f)
$$

The two types of propagators $q_{j}(r, s)$ and $q_{j}^{\dagger}(r, s)$ (with $j=\mathrm{A}, \mathrm{B}$ ) are solutions of the modified diffusion equation

$$
\frac{\partial q_{j}(r, s)}{\partial s}=\left(\frac{R_{g}}{\ell_{0}}\right)^{2} \nabla^{2} q_{j}(r, s)-\omega_{j}(r) q_{j}(r, s)
$$


with the initial condition $q_{A}(r, s=0)=q_{B}(r, s=0)=1, \quad q_{A}^{\dagger}(r, s=0)=q_{B}(r, 1-f)$ and $q_{B}^{\dagger}(r, s=0)=q_{A}(r, f)$. This diffusion equation is solved using reflecting boundary conditions at the two confining surfaces $(z=0$ and $z=L): \mathrm{d} q /\left.\mathrm{d} r\right|_{z=0}=0$ and $\mathrm{d} q /\left.\mathrm{d} r\right|_{z=L}=0$, while periodic boundary conditions are used in the perpendicular direction.

The top surface with parallel and elongated grooves is modeled as an impenetrable boundary for the polymer chains. In practice, this is done by assigning a large value to the local surface field, $u_{A}(r)=u_{B}(r)=-20$, for all points inside the grooves. In addition, on the whole top surface we include a weak attractive field $(u=0.02)$, which takes into account the preferred surface interaction with the A block.

We use the same numerical method as that in our previous work [25] to solve these selfconsistent equations. Fields and densities were calculated on a grid with a spatial resolution of $\Delta \approx \ell_{0} / 10$ or $\Delta \approx \ell_{0} / 20$ for the three- and two-dimensional grids, respectively. First, we guess an initial set of values for the $\left\{\omega_{j}(r)\right\}$ auxiliary fields. Then, through the diffusion

equations we calculate the propagators, $q_{j}$ and $\left\{q_{j}^{\dagger}\right\}$, and from Eq. (5) and Eq. (6) we calculate the monomer volume fractions $\left\{\phi_{j}\right\}$. Next, a new set of values for $\left\{\omega_{j}(r)\right\}$ is obtained through Eq. (3) and Eq. (4) and this procedure can be iterated until convergence is obtained by some conventional criterion. More details are given in Ref. [25].

\section{RESULTS}

Figure 2 shows a top view of SEM and AFM images of a $\mathrm{PS}_{52 \mathrm{~K}}$-b-PMMA $\mathrm{P}_{52 \mathrm{~K}}$ thin film after nanoimprinting with a mold made of a series of parallel grooves [Fig. 1]. From the images, it can be seen that the lamellar phase is indeed oriented perpendicular to the bottom substrate. Moreover, in Fig. 2(a) the SEM image indicates that the thicker sections of the BCP film exhibit lamellae that are nicely oriented in a parallel fashion along the groove vertical walls (the $y$-direction), while the thinner sections of the film are too thin to be visualized by the SEM technique. The same in-plane ordered lamellae are shown as an enlargement in Fig. 2(b), whereas in Fig. 2(c) the region close to the groove vertical wall is visualized by an AFM technique. The AFM image indicates a different in-plane ordering of the thick and thin sections of the BCP film. While the thick section (right) shows well ordered lamellae of periodicity very close to the bulk one, $\ell_{0}$, which are aligned by the wall, the lamellae of the thin section (left) of the film are less oriented and contain some defects. This observation can be attributed to the preferred interaction of the mold vertical walls with one of the two BCP blocks. The lamellar film to the right of the wall is thicker in the $z$-direction (height $L_{h}$ as in Fig. 1(b)) and is in contact with the vertical groove wall, while the film section on the left has a smaller height $\left(L_{l}\right)$, and the wall has no direct influence on it.

In order to further understand these NIL results, we complement the experiments 

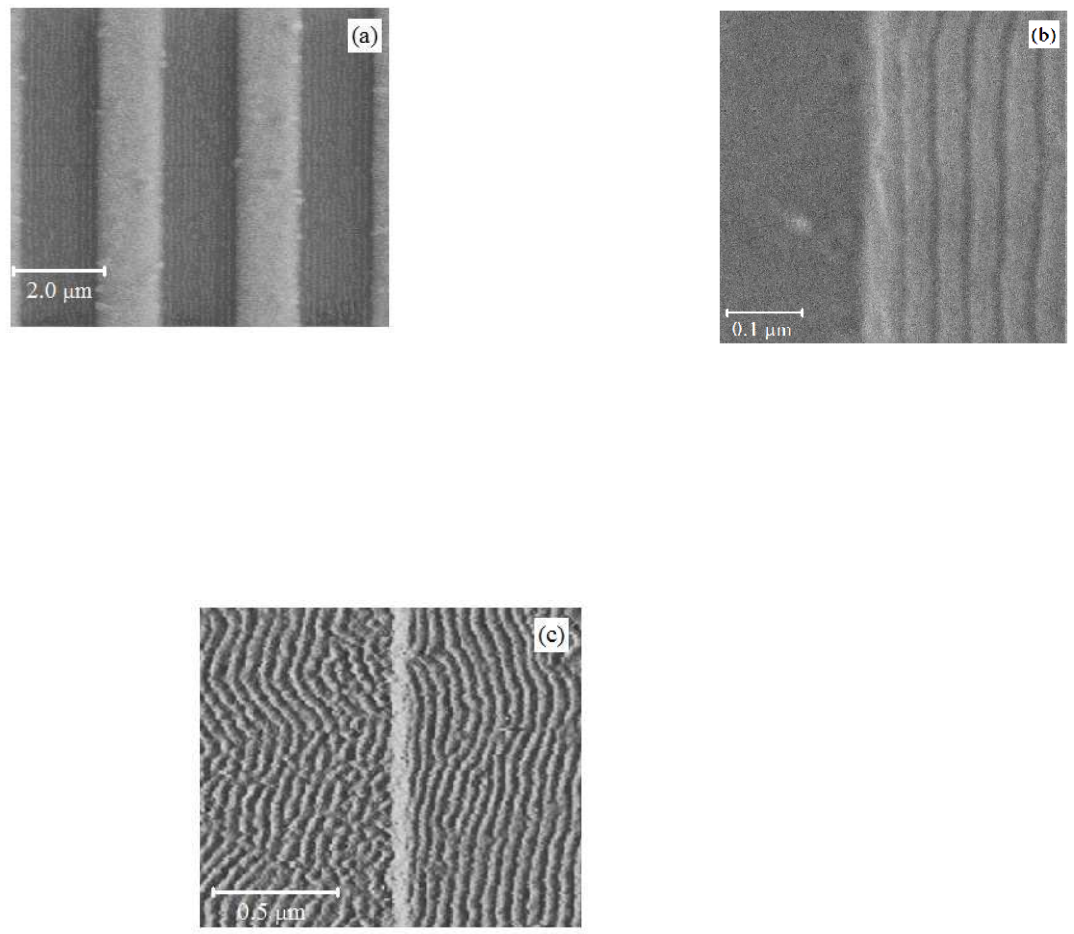

FIG. 2: (a) Top view of a SEM image of the BCP film after nanoimprinting with a NIL mold with $\omega_{l}=\omega_{h}=1.5 \mu \mathrm{m}$. The groove height is $L_{h}-L_{l}=50 \mathrm{~nm}$. The perpendicularly lying BCP lamellae are further oriented along the groove long axis. (b) An enlargement of (a) where several lamellae (left) are well ordered in-plane. (c) An AFM top view of an enlarged section of the BCP film close to thin-thick boundary (the middle vertical line). To the right, the BCP film is thicker (thickness $L_{h}$ ) and is ordered by the groove vertical wall, whereas to the left, the BCP is thinner (thickness $L_{l}$ ) and less ordered.

with self-consistent field (SCF) theory calculations performed on symmetric BCP lamellar phases [20-24]. The NIL setup is modeled by BCP lamellae having a natural periodicity $\ell_{0}$, and confined in the $z$-direction between a flat and neutral bottom surface $(u=0)$ at $z=0$ and a topographical varying surface (the top mold), as is shown schematically in Fig. 1(b). The top surface has the form of elongated grooves (in the $y$-direction) of square cross section (in the $x$-direction). The down-pointing indentations (fingers) have a width of $\omega_{l}$ separated by grooves with a cross-section width $\omega_{h}$. The BCP film thickness measured with respect to the $z=0$ surface varies between $L_{h}$ inside the grooves and $L_{l}$ in between the grooves [Fig. 1(b)]. The mold preferential interaction toward one of the two blocks is modeled by 
an overall non-zero surface field, $u>0$.
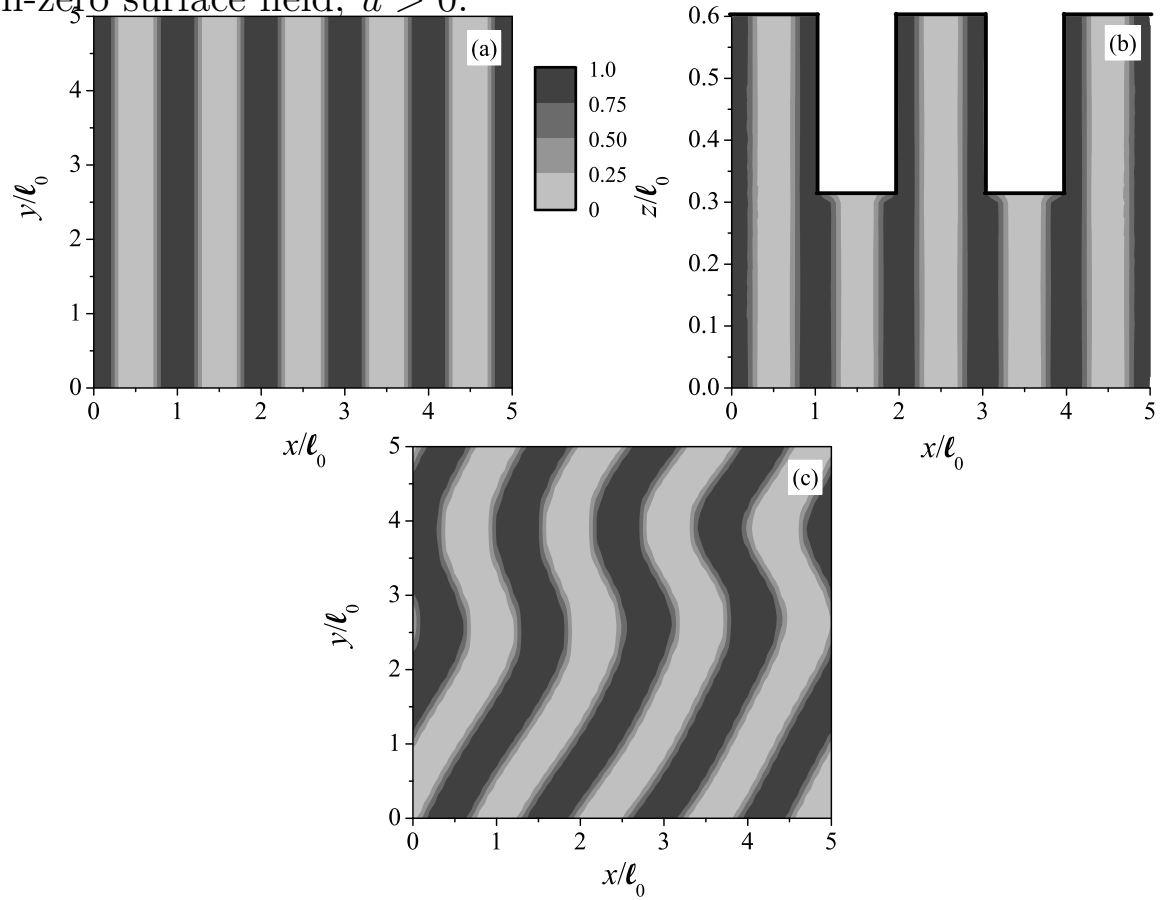

FIG. 3: Calculated 3d lamellae in a BCP film of size: $L_{x} \times L_{y} \times L_{z}=10 \ell_{0} \times 10 \ell_{0} \times 0.6 \ell_{0}$ and $N \chi=25.0$. The system parameters for the NIL are: $u=0.02, d=4 \ell_{0}, \omega_{l}=\omega_{h}=2 \ell_{0}, L_{h}=0.6 \ell_{0}$ and $L_{l}=0.3 \ell_{0}$. (a) Top-view cut at $z=0.3 \ell_{0}$ and (b) side cut at $y=5 \ell_{0}$ of the simulated NIL setup. The perfect in-plane order seen in (a) is accompanied by a perfect perpendicular order in (b). For comparison, a top-view cut at $z=0.3 \ell_{0}$ is presented in (c) for a similar system bound by two neutral and flat surfaces at $z=0$ and $z=0.6 \ell_{0}$. It shows several in-plane defects embedded into the perpendicularly oriented lamellar phase.

We performed several three dimensional (3d) SCF calculations to shed light on the film in-plane ordering. The $3 \mathrm{~d}$ system size is $L_{x} \times L_{y} \times L_{z}=10 \ell_{0} \times 10 \ell_{0} \times 0.6 \ell_{0}$, where $L_{z}$ is set to be less than one BCP periodicity in accord with the experiments (Fig. 2). In our 3d $\mathrm{SCF}$ calculations, a gradual temperature quench is performed. The starting temperature is above the order-disorder temperature (ODT), $N \chi_{c} \simeq 10.5$; hence, inside the BCP disordered phase. The temperature is then gradually decreased (or, equivalently, the value of $N \chi$ is gradually increased) until the system reaches $N \chi=25$, which is well below the ODT.

With the simulated NIL mold, a perfect perpendicular lamellar structure is found and is shown in Fig. 3(a) as a top-view cut at $z=0.3 \ell_{0}$, and in $3(\mathrm{~b})$ as a side cut at $y=5 \ell_{0}$. Note that in the calculation, Fig. 3(a), the thinner and thicker sections of the film are equally ordered, whereas in the experiments, Fig. 2(c), the in-plane ordering is not as good for the 

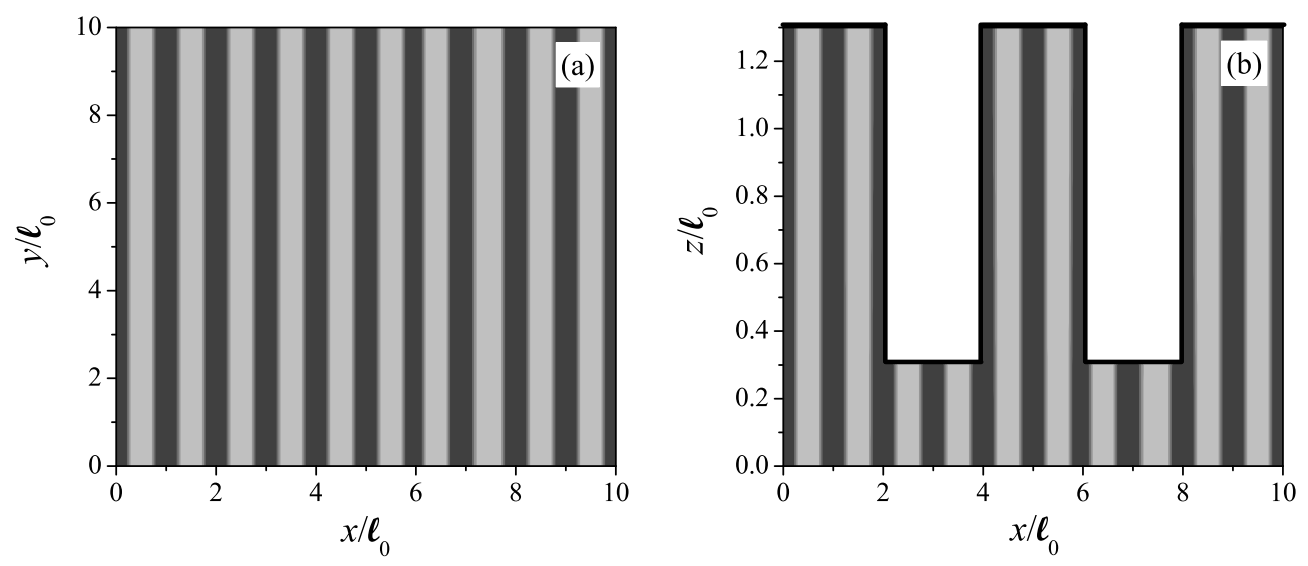

FIG. 4: Calculated 3d lamellae in a BCP film of size: $L_{x} \times L_{y} \times L_{z}=10 \ell_{0} \times 10 \ell_{0} \times 1.3 \ell_{0}$. All other parameters are the same as in Fig.3(a). (a) Top-view cut at $z=0.3 \ell_{0}$ and (b) side cut at $y=5 \ell_{0}$ of the simulated NIL setup. The perfect in-plane order seen in (a) is accompanied by a perfect perpendicular order in (b).

thinner section of the film (left side of the figure) as compared with the thicker film section on the right side. This lack of in-plane ordering in the experiments might be due to lack of equilibration or shear effects at the walls, and more detailed investigations are needed to further clarify this point.

As a further check, we compare the NIL setup with a BCP film confined between two neutral and flat surfaces. As can be seen in Fig. 3(c), a perpendicular lamellae structure arises with no in-plane ordering when the same gradual temperature quench process is repeated for the neutral and flat surfaces. It is clear that there are many in-plane defects in this case, in contrast with the NIL mold that induces a strong in-plane ordering.

We show another 3d calculation in Fig. 4, for which all the parameters are the same as in Fig. 3 (a) except that we modify the $L_{h}$ value to be $L_{h}=1.3 \ell_{0}$. We still obtain the perfect perpendicular lamellae for this thicker BCP film, which is consistent with the phase diagram of Ref [25]. We conclude that the value of $L_{h}$ is not strictly limited, but can have a range of values that will result in a perfect BCP perpendicular lamellar phase.

Another important question we addressed is the stability of the perpendicular phase for wider grooves (larger $\omega_{h}$ ). To further explore this issue we complemented the $3 \mathrm{~d}$ calculations by $2 \mathrm{~d}$ ones where much larger lateral system sizes (up to $L_{x} \times L_{z}=1.3 \ell_{0} \times 80 \ell_{0}$ ) can be simulated. Although the two-dimensional calculation cannot infer on the degree of in-plane ordering and defects, it is of value because it shows the effects of groove width on the stability of the perpendicular BCP lamellae. In Fig. 5(a), an ordered perpendicular phase is found for the NIL setup with $d=20 \ell_{0}$. This figure should be compared with the $3 \mathrm{~d}$ calculations 

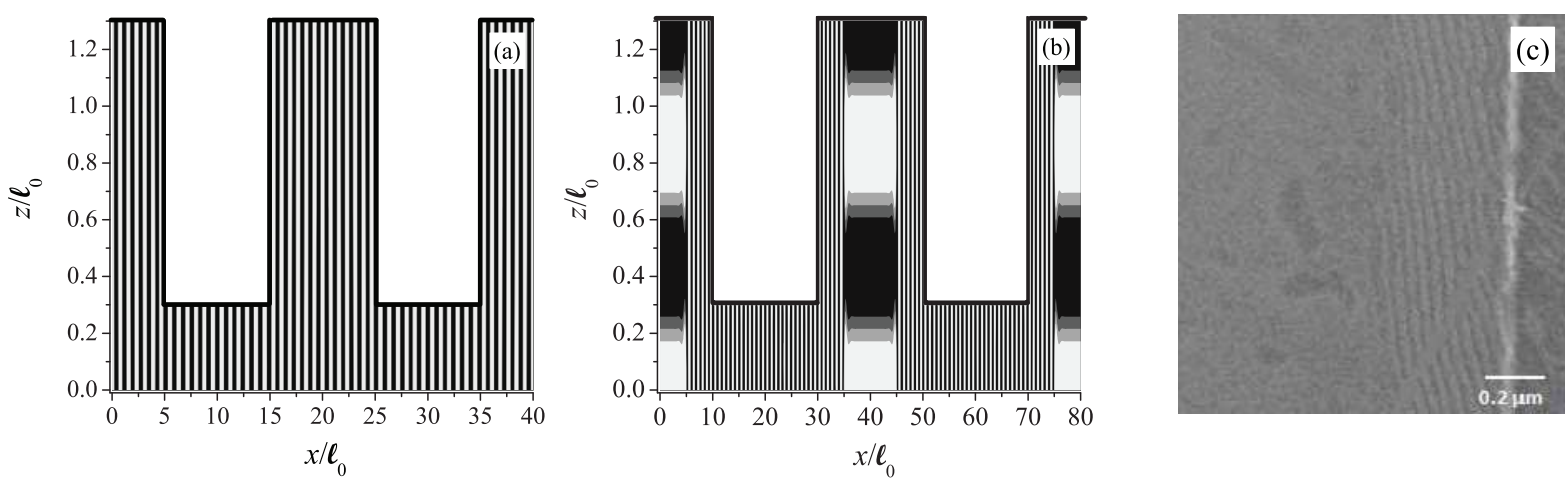

FIG. 5: (a) Calculated 2d structures of BCP lamellae in the $x-z$ plane for $N \chi=20.0$. The system parameters are $u=0.02, d=20 \ell_{0}, \omega_{l}=\omega_{h}=10 \ell_{0}, L_{h}=1.3 \ell_{0}$ and $L_{l}=0.3 \ell_{0}$. (b) Same as (a) but with an increased groove periodicity $d=40 \ell_{0}$ with $\omega_{l}=\omega_{h}=20 \ell_{0}$. (c) Top view of a SEM image of a $\mathrm{PS}_{52 \mathrm{~K}}$-b-PMMA $52 \mathrm{~K}$ film after NIL. For groove width of about $20 \ell_{0}$ the perpendicular lamellar phase persists only near the groove edge (white vertical line on the right). In the region far from the groove edge, the lamellae probably change their orientation to one that is parallel to the substrate. The bar scale is $0.2 \mu \mathrm{m}$.

as shown in Fig. 3(a) for much narrower grooves of $d=2 \ell_{0}$.

The NIL setup has some limitations as can be seen from our calculations and through preliminary experimental results. If we increase the groove periodicity (scaling up both $\omega_{h}$ and $\left.\omega_{l}\right)$, the perfect perpendicular lamellae do not persist. Instead, the film breaks up into a mixed morphology, combining a perpendicular phase close to the groove wall with a parallel phase induced by the horizontal section of the top surface. This can be seen in Fig. 5(b), where all parameters are the same as in 5(a) beside a larger groove periodicity, $d=40 \ell_{0}$. These findings are in accord with the experimental ones shown in Fig. 5(c), where SEM images demonstrate the loss of the perpendicular lamellae, for large enough groove periodicity, after distances of about $10 \ell_{0}$ from the groove wall.

\section{DISCUSSION \& CONCLUSIONS}

We address in this article the influence of nano-patterning of surfaces on the orientation and alignment of lamellar phases of BCP films, using a NIL technique to produce superior perpendicular ordering. The main goal of the NIL is to be able to use surface features on length scales larger than the BCP natural periodicity $\ell_{0}$ in order to reduce the cost of expensive surface preparation treatments. 
The effect of the NIL setup on BCP in-plane ordering is clearly demonstrated both in the experiments and in the modeling. Without the NIL mold, it is possible to obtain perpendicular lamellae but with many in-plane defects which cannot be annealed away. However, with the NIL mold, wetting of the vertical groove wall induces perfect perpendicular ordering with minimal amount of defects, over large lateral distances.

In our model the perfect perpendicular order is induced by a small surface field $u=0.02$, chosen to agree with PS-PMMA experimental setup, where the relative difference in the surface tension between the two blocks and the surface is about $1 \%$. The value of $u$ is limited by two opposing trends. On the one hand, $u$ should be large enough so that one of the two blocks would wet the groove vertical wall. On the other hand, $u$ should be small enough in order not to interfere with the overall perpendicular ordering.

Another conclusion from the present study is that the NIL groove periodicity cannot be much larger than $\ell_{0}$; otherwise the BCP perpendicular order is lost [see Fig. 5(b) and (c)], where the perpendicular order is lost for about $d \simeq 20 \ell_{0}$.

Our SCF calculations are in good agreement with the presented experiments, and provide some insight into the conditions needed to obtain perpendicular lamellar phases with minimal amount of in-plane defects. We hope that in the future more detailed three-dimensional calculations as well as careful investigations of film rheology will shed more light on the fundamental behavior as well as applications of BCP films in presence of nano-patterned surfaces.

\section{Acknowledgements}

The help of S. Niedermayer in sample preparation is gratefully acknowledged. We would like to thank the Triangle de la Physique, France (POMICO project No. 2008-027T) for supporting the visits of DA and XM to Saclay. This work was supported in part by the U.S.-Israel Binational Science Foundation under Grant No. 2006/055, the Israel Science Foundation under Grant No. 231/08, the Center for Nanoscience and Nanotechnology at Tel Aviv University and the CEA (France) under programs "Chimtronique" and "Nanosciences".

[1] Bang, J.; Jeong, U.; Ryu, D. Y.; Russel, T. P.; Hawker, C. J. Adv. Mater. 2009, 21, 4769.

[2] Black, C. T.; Ruiz, R.; Breyta, G.; Cheng, J. Y.; Colburn, M. E.; Guarini, K. W.; Kim, H. C.; Zhang, Y. IBM J. Res. \& Dev. 2007, 51, 605.

[3] Kim, H.-C.; Park, S.-M.; Hinsberg, W. D. Chem. Rev. 2009, 110, 146. 
[4] Leibler, L. Macromolecules 1980, 13, 1602.

[5] Mansky, P.; Liu, Y.; Huang, E.; Russell, T. P.; Hawker, C.-J. Science 1997, 275, 1458.

[6] Liu, P. H.; Thébault, P.; Guenoun, P.; Daillant, J. Macromolecules 2009, 42, 9609.

[7] Ham, S.; Shin, C.; Kim, E.; Ryu, D. Y.; Jeong, U.; Russell, T. P.; Hawker, C. J. Macromolecules 2008, 41, 6431.

[8] Han, E.; Stuen, K. O.; La, Y.-H.; Nealey, P. F.; Gopalan, P. Macromolecules 2008, 41, 9090.

[9] Ruiz, R.; Kang, H. M.; Detcheverry, F. A. Dobisz, E.; Kercher, D. S. Albrecht, T. R.; de Pablo, J. J.; Nealey, P. F. Science 2008, 321, 936.

[10] Stoykovich, M.; Müller, M.; Kim, S.; Solak, H.; Edwards, E.; de Pablo, J. J.; Nealey, P. Science 2005, 308, 1442.

[11] Ruiz, R.; Sandstrom, R. L.; Black, C. T. Adv. Mater. 2007, 19, 587.

[12] (a) Segalman, R.; Yokoyama, H.; Kramer, E. Adv. Mater. 2001, 13, 1152. (b) Stein, G. E.; Lee, W. B.; Fredrickson, G. H.; Kramer, E. J.; Li, X.; Wang, J. Macromolecules 2007, 40, 5791.

[13] Park, S. M.; Stoykovich, M. P.; Ruiz, R.; Zhang, Y.; Black C. T.; Nealey, P. E. Adv. Mater. 2007, 19, 607 .

[14] (a) Cheng, J. Y.; Ross, C. A.; Thomas, E. L.; Smith, H. I.; Vancso, G. J. Appl. Phys. Lett. 2002, 81, 3657. (b) Chuang, V. P.; Cheng, J. Y.; Savas, T. A.; Ross, C. A. Nano Lett. 2006, 6,2332 .

[15] (a) Sundrani, D.; Sibener, S. J. Macromolecules 2002, 35, 8531. (b) Sundrani, D.; Darling, S. B.; Sibener, S. J. Nano Lett. 2004, 4, 273.

[16] Chou, S.; Krauss, P.; Renstrom, P. Appl. Phys. Lett. 1995, 67, 3114.

[17] Hu, Z.; Baralia, G.; Bayot, V.; Gohy J.; Jonas, A. Nano Lett. 2005, 5, 1738.

[18] Li, H.; Huck, W. Curr. Op. Solid State Mat. 2002, 6, 3; Li H.; Huck, W. Nano Lett. 2004, 4, 1633.

[19] Kim, S.; Lee, J.; Jeon, S.-M.; Lee, H. H.; Char K.; Sohn, B.-H. Macromolecules 2008, 41, 3401.

[20] Matsen, M. W.J. Chem. Phys. 1997, 106, 7781.

[21] Petera, D.; Muthukumar, M. J. Chem. Phys. 1998, 109, 5101.

[22] Pereira, G. G.; Williams, D. R. M. Europhys. lett. 1998, 44, 302.

[23] Geisinger, T.; Mueller, M.; Binder, K. J. Chem. Phys. 1999, 111, 5241. 
[24] Tsori Y.; Andelman, D. Eur. Phys. J. E. 2001, 5, 605.

[25] Man, X. K.; Andelman, D.; Orland, H. Macromolecules 2010, 43, 7261. 
for Table of Contents use only

\section{Organization of Block Copolymers using NanoImprint Lithography: Comparison of Theory and Experiments}

Xingkun Man, David Andelman, Henri Orland, Pascal Thébault, Pang-Hung Liu,Patrick Guenoun, Jean Daillant, Stefan Landis 